\title{
Online Voice Based Learning Environment for English Language
}

\author{
R.S.P Ranasinghe, A.H.M Weerathunga, K.B.P Kariyawasam, H.L Premaratne, \\ K. P. Hewagamage \\ University of Colombo School of Computing, Sri Lanka
}

\begin{abstract}
Introducing of an online tool that could be used to enhance the language competency of a person is described in this paper. It entails how to develop the communication ability of a person using a computer guided software as well as the help of an online mentor. This research is based on designing an "Online Voice Based Learning Environment for English Language (VBLMS)" which consists of three components. They are, the Pronunciation training tool to assist people to improve their pronunciation, Voice based online forum, enables sharing of knowledge among each other and Online English language learning room which enables the people to join online classes and get help of online mentors and share the knowledge in real time. Implemented solution was tested with online users and the results on the user study shows that the productivity of Virtual Classrooms compared to Traditional Classrooms. Therefore the solution directly facilitates the major aspects of language learning, which are Reading, Writing and speaking with proper pronunciation.
\end{abstract}

\section{Introduction}

Effective communication plays a key role in academic, professional and personal success. Communication skills are learned and acquired through practice. Effective communication involves multiple components, including non-verbal communication, effective listening, knowing how to draw someone out, assertiveness, conflict resolution and anger management, etc.

Students who are in developing countries should have to pass exams such as TOEFL (Test of English as a Foreign Language), IELTS (International English Language Testing System), etc. to attend universities in developed countries to continue with their studies. In order to get through such exams, one need to competent in all three major aspects of a language, which are Reading, Writing, Creative speaking with proper pronunciation. Generally they have to use learning material such as CDs, books, and hand outs to train themselves. In addition to these learning materials, there are many forums and online Learning Management Systems (LMS) on the internet for people to share their knowledge regarding language competencies. But most of them are text based forums, which would only assist reading \& writing skills.

Therefore it will be more effective and attractive if people can share their knowledge using their own voice. An online voice based tool would serve the purpose really well. Such a tool would assist people in improving their assertiveness while reducing their shyness to speak in public.

In achieving interactivity in distance learning, it is essential to have a real-time voice communication tool on the web. This will enable groups of people such as students and teachers to participate in discussion groups through synchronous (real-time) and asynchronous chat sessions. This will additionally help the schools and the universities for their teaching purposes as well.

The system is an enhancement for e-learning because it improves the e-learning conception through the introduction of voice based interaction. The ultimate output of this project will be "an online voice based learning environment" that would assist people to enhance their communication skills through collaborative learning methods.

The concept of distance learning will be used to good effect by the project, creating a whole mutually-supportive voice based network for training on communication and language competencies.

The main objectives of this project are to

- Build an online system which can assist people to improve their pronunciation.

- Build an online voice based forum, which can facilitate to share ideas among web-based communities.

- Build a virtual learning environment, an "Online Class Room", which is based on voice format.

The way of combining these components to a single architecture, the technologies used, and the 
evaluation \& conclusion will be discussed in detail in rest of the document.

\section{Related Work}

We are focusing our review referring to previous work in this field which were carried out on implementing pronunciation training tools, voice based forums and community websites and also virtual classrooms and other related work. We mine knowledge on those areas covering the existing technologies, software tools for English language learning, English language learning concepts, voice base human computer interaction, open source resources that can be useful, etc. The review is carried out based on the prior researches and findings done by the researchers around the world. The review will further consider design methodologies to facilitate the production and use of online material resources such as voice forums and online class rooms to aid pronunciation and competencies in second language learning.

\subsection{Second Language Learning}

The field of second language acquisition (SLA) is concerned with the study of how a new language other than the native mother tongue is learned. It includes reading, writing, grammar, vocabulary, listening, speaking and culture. In language learning and speaking, pronunciation forms an important part of the communication process. Poor pronunciation not only distracts the listener, it also makes him difficult to understand the message and may result in the speaker being negatively categorized in a social context. For many adult learners of a foreign language the biggest hurdle is speaking the target language. Some research argues that the teaching of pronunciation makes no difference, while other research has shown that a "noticeable difference" can be made through teaching if certain rules are followed.

A Europe-wide survey carried out by the European Centre for Modern Languages found that although the communicative theoretical approach which has a strong emphasis on oral activities is widely applied in second language learning throughout Europe, learners complained that too little time was being spent on speaking. According to students, the main objective for undertaking a second language is being able to speak the language. This is frequently underreported in course design. It is estimated that the vast majority of those who attend foreign language courses in the United States do not acquire functional competence using the language.

As stated earlier, research on learning a second language indicates that there are conflicting views with regard to a suitable approach for phonological (sounds in words) instruction. However, there is less research into pronunciation than into syntax or discourse, and the belief that the reason for this is for many learners intelligibility in spontaneous speech is a sufficient goal.

\section{i. Microsoft Speech Application Programming} Interface. The Speech Application Programming Interface(SAPI) is an API developed by Microsoft to allow the use of speech recognition and speech synthesis within windows applications.

To date, a number of versions of the API has been released, which has shipped either as part of a Speech SDK, or as part of the Windows OS itself. Applications that use SAPI include Microsoft Office, Microsoft Agent and Microsoft Speech Server. In general, all versions of the API have been designed such that a software developer can write an application to perform speech recognition and synthesis by using a standard set of interfaces, accessible from a variety of programming languages.

\section{ii. Using Online Resources to Aid Pronunciation.} The field of computer assisted language learning (CALL) has expanded rapidly over recent years. The widening provision of broadband and affordable computer hardware and software means, that a growing number of learners have access to online learning material and resources. It is important to note that any technology is only as good as its use. That is to say that there are a number of online resources already available, but unless they are used properly they will not have a useful impact on learning. A pattern throughout the literature was a caution regarding the possible novelty value of computer assisted/ online multimedia tools, clouding a true evaluation of its usefulness. It was found that more than half the class time was spent getting to the lab, logging on and setting up. However, the user was very positive about the activity thereafter. The view that computer assisted/ multimedia/ online resources were generally of benefit to language learning, and pronunciation was held by the majority throughout this literature review.

After decades of being treated by much of the language education community as an afterthought, second language pronunciation is benefiting from renewed focus from educators and researchers. As emphasis on conversational or communicative language skills increases in academic and professional environments, more pronunciation and listening comprehension materials are finding their way to market.

Employing computer-based pronunciation programs can benefit students and teachers in that such programs make it possible to address individual problems, allow students to work at their own tempo, and may lead to reduction in classroom anxiety. Overall, CAPT allows for increased practice time to "more closely approach the advantages of total 
immersion learning”. Success in the language lab, or any self-study environment, requires appropriate feedback. Early forms of computer-based instruction featured aids such as head diagrams and visual models - aids that have questionable educational benefit. Later forms of CAPT included automatic speech recognition (ASR) features such as spectrograms or oscillograms that look impressive but may be un-interpretable to learners and may be better suited to practice than learning. The next generation of CAPT features technology that allows learners to hear their own voices, in real time, modulated into the frequency of the target language. Rather than relying on visual models or graphical representations, students learn to listen and speak in a second language by following the sound of their own voices. Technologies such as Speed APIs close the gap between listening and speaking, removing the barriers to intelligibility and comprehension.

\subsection{Voice Forum}

The key findings and innovations related to voice forums are discussed below. It contains the research and work that has been done so far in this stream of voice based forums. The currently available voice forums are listed below with detailed insight into the functionalities of those forums.

Considering the typical forum procedure, it was the public space in the middle of a Roman city. It was a gathering place of great social significance. It was often the scene of diverse activities, including political discussions, meetings, et cetera. So in today's world Forum means a place or an event which facilitates people to get together and exchange their knowledge and ideas.

Wikitionary defines a forum as a place for discussion, a gathering for the purpose of discussion, a form of discussion involving a panel of presenters and often participation by members of the audience, an internet message board where users can post messages regarding one or more topics of discussion. Considering the available voice forums, Internet Shout is a social networking site which is based on verbal communication. The site purports to be the world's first ever purpose built voice based forum, allowing mouthy users to record sound clips to the website and start conversations, participate in ongoing conversations or, if you're shy, just listen in.

Also Chinswing has launched a public beta of a new voice-based message board that combines features of podcasting, text message boards, and live voice chat. By adding voice messages to topical discussions, users can share similar interests with others.

NanoGong is an applet that can be used by someone to record, playback and save their voice, on a web page. When the recording is played back the user can speed up or slow down the sound without changing it.

Also the Wimba Voice Tools allow students and teachers to learn and teach in the most natural way of all - with voice. The faculty can easily add synchronous and asynchronous vocal collaboration into their course management systems. All a learner or instructor needs is a computer with a microphone and speakers, and an internet connection.

\subsection{Online Language Learning Room}

The previous work related to Online Language Learning Room that has been done so far is discussed below by analyzing and reviewing the literature.

Rather than a lecture, online instructional approach involves learners who work from individual computers and continually interact as they respond to instructor questions, discuss issues in "breakout rooms", and add their ideas to a group online chart.

In relation to the term 'virtual classroom', the word virtual can be understood as, "being actively connected to a network or computer system; usually being able to interactively exchange data, commands, and information". When we combine this definition with the traditional idea of a classroom being a physical room containing an instructor and students who learn through face-to-face interaction, we are able to derive a suitable definition of the term. A virtual classroom, therefore, is a learning environment that exists solely in the form of digital content that is stored, accessed, and exchanged through networked computer and information systems. Everything in a virtual classroom occurs in a non-physical environment; students access the classroom by connecting to the internet, rather than travelling to a real, physical classroom. The students may not even be in the same country as the instructor or teacher; the nature of virtual classrooms means that, in terms of access, the geographic location of students is not an issue.

In recent times, there is a growing number of online courses being developed using the social constructivist approach, particularly the social constructivist approach, which encourages students to collaborate and reflect to co-construct new understandings through uniquely personal experiences in the context of inquiry. From the social constructivist paradigm, one of the potentials of the Web is its ability to encourage interactions and students' involvement in the learning environment.

WiZiQ Live Class enables you to schedule your live online classes for your course students. A single time sign-up on WiZiQ allows you to set up these live classes. You can schedule your live class for any date and time in your time zone and for any number of students. 


\section{Requirement Gathering}

A user assessment was carried out to determine the participants' idea about the VBLMS as a learning tool. Before starting the project we did a survey to find out the feasibility of this project and their ideas about the project using an Online Questionnaire. The questions basically targeted the main three components of the VBLMS, Pronunciation Training Tool, Voice forum and Online Class Room.

All the questions are formed in a way which is extorting the need and ideas of the responders about the system. We used the knowledge we gathered during the Literature Review earlier, in the process of formulating questions. Also we took some available questioners as examples. Also we used some ideas of professionals. In our questioner we have used only quantitative questions with closed answers. The order of the questions in the questionnaire generally follow a template where it start with more basic, general questions, move on to more in-depth and demanding questions and then end with simple, easy to answer questions.

When all the preparations have been made it is time to send the questionnaire to the respondents. We prepared an online blog at blogspot.com as follows: http://virtuallanguageclass.blogspot.com/. We published the Introduction chapter of the project in that blog for the knowledge of the responders. We placed the questioner beside the introduction chapter. In the questioner we only count the number of hit under a particular answer.

After preparing the blog, we mailed our blog link to our target users through e-mails and also send it to social networks such as facebook and hi5. They are distributing the massage in the virus selection method. Soon it will be distribute among a large set of responders. However, it is very rare that everyone who has received a questionnaire answers it. The answer frequency is nothing more than a percentage that shows how many has answered. Of course we are aiming to have as many votes to the questionnaire as possible. To increase the answer frequency, we have used some methods,

- We have send reminders,

- Try to keep down the number of questions in the questionnaire,

- Avoiding sensitive questions if possible

- Finding and correcting errors in the questionnaire before they are distribute.

Two hundred and fourteen (214) participants joined to the questionnaire and the results analysis is as follows;

- People like to take online classes, they prefer it more than the traditional classes, and they were looking forward to experiencing it.
- They cannot imagine the experience before participating to such a class, but they feel it might be enjoyable and have a good experience from it.

- They think it's a great idea if this concept could be implemented.

- If the voice forums concept is feasible it would be great because it will help to capture more information such as ideas and emotions in a few seconds than text based forums.

- Most participants like to practice in pronunciation sessions with the help of a professional tutor, but they also like to compare it with a computer based verification tool as well.

- They prefer to have an experience of a portal which is an integration of online classroom and a voice forum.

From the observations gained by the online questionnaire, we realized that the VBLMS could be a good online solution for the people who are waiting for enhance their communication ability as well as share knowledge and ideas among each other, without a geographical barrier.

In the next step we designed and implemented a prototype of the system according to the observed requirements in the survey. After that, we implemented the prototype in the web and gave access to the target group. We gathered their ideas through a questioner to fine-tune the system and its requirements.

\section{Design of VBLMS}

We have analyzed the requirements that we gathered in section 3 , and identified requirements for the VBLMS. Based on that, we have identified and designed the use case diagrams. First we designed a high level use case diagram and then three other use case diagrams covering the functionalities of three main components. Then we drew a class diagram that will show the relationship between the identified classes. Based on both use case and class diagrams we drew relevant sequence diagrams.

We have analyzed the requirements of the VBLMS closely and following are the functional and non-functional requirements that we have identified for each component of the VBLMS.

\subsection{Functional Requirements}

\section{i. For Pronunciation Training Tool}

A. Need to calculate the confidence level of pronunciation automatically

B. Need to store the pronounced speech in the server. C. Need to play the stored speech. 


\section{ii. For Voice Forum}

D. Need to create a post in a forum by recording a speech.

E. Need to convert the recording speech in to text and store with the post.

F. Need to play the speech in the post.

G. Need to search for keywords in stored text in posts.

\section{iii. For Online Language Learning Room}

H. Teacher and students should be able to communicate with each other real-time using their voice.

I. Need to search for keyword in the classes.

J. Need to share materials online.

K. Need to have a shared writing space.

iv. Other

L. User Management

\subsection{Non-Functional Requirements}

$\begin{aligned} \text { i. } & \text { Usability } \\ \text { ii. } & \text { Performance } \\ \text { iii. } & \text { Correctness } \\ \text { iv. } & \text { Availability } \\ \text { v. } & \text { Reliability } \\ \text { vi. } & \text { Security }\end{aligned}$

Following table 1 shows the relationship between functional and non-functional requirements. Nonfunctional requirements are weighted according to the importance of them to the VBLMS. And the total weight is calculated for each functional requirement. Higher the total is, higher the criticality of that particular function to VBLMS. The functions that have higher criticality will be implemented as early as possible.

Table 1. Requirement Analysis Matrix

\begin{tabular}{|c|c|c|c|c|c|c|c|c|}
\hline \multirow{14}{*}{ 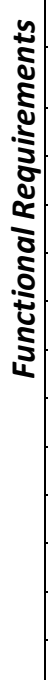 } & \multicolumn{7}{|c|}{ Non-Functional Requirements } & \multirow{2}{*}{$\begin{array}{l}\text { Total } \\
\text { Weight }\end{array}$} \\
\hline & & $\begin{array}{c}i \\
W=6\end{array}$ & $\begin{array}{c}\text { ii } \\
W=6\end{array}$ & $\begin{array}{c}\mathrm{iii} \\
\mathrm{W}=5\end{array}$ & $\begin{array}{c}\text { iv } \\
W=4\end{array}$ & $\begin{array}{c}v \\
w=4\end{array}$ & $\begin{array}{c}v i \\
W=3\end{array}$ & \\
\hline & A & & $\checkmark$ & $\checkmark$ & & & & 11 \\
\hline & $\mathrm{B}$ & & $\sqrt{ }$ & & & $\sqrt{ }$ & & 10 \\
\hline & C & $\checkmark$ & $\checkmark$ & & $\checkmark$ & $\checkmark$ & & 20 \\
\hline & $\mathrm{D}$ & $\checkmark$ & $\checkmark$ & & & $\sqrt{ }$ & & 16 \\
\hline & $E$ & & & $\checkmark$ & & $\checkmark$ & & 9 \\
\hline & $\mathrm{F}$ & $\checkmark$ & $\checkmark$ & & $\checkmark$ & $\checkmark$ & & 20 \\
\hline & G & $\checkmark$ & $\checkmark$ & $\checkmark$ & & & & 17 \\
\hline & $\mathrm{H}$ & $\checkmark$ & $\checkmark$ & & $\checkmark$ & $\checkmark$ & & 20 \\
\hline & 1 & $\checkmark$ & $\checkmark$ & $\checkmark$ & & & & 17 \\
\hline & $\mathrm{J}$ & $\checkmark$ & & & $\sqrt{ }$ & $\sqrt{ }$ & & 14 \\
\hline & $\mathrm{K}$ & $\sqrt{ }$ & $\checkmark$ & & $\checkmark$ & & & 16 \\
\hline & $\mathrm{L}$ & & & & & $\checkmark$ & $\checkmark$ & 7 \\
\hline
\end{tabular}

\subsection{System Architecture}

The VBLMS system is built based on a client and server architecture. After submitting the authentication information to the server via a web browser the user authorized to access the web server and request for the learning materials. The figure 1 illustrates how the VBLMS is functions in a real time.

The system provides web content developers the opportunity to add audio capability to a website. For example, a course web page can contain a voice board which allows students to discuss with the teachers verbally on the Web.

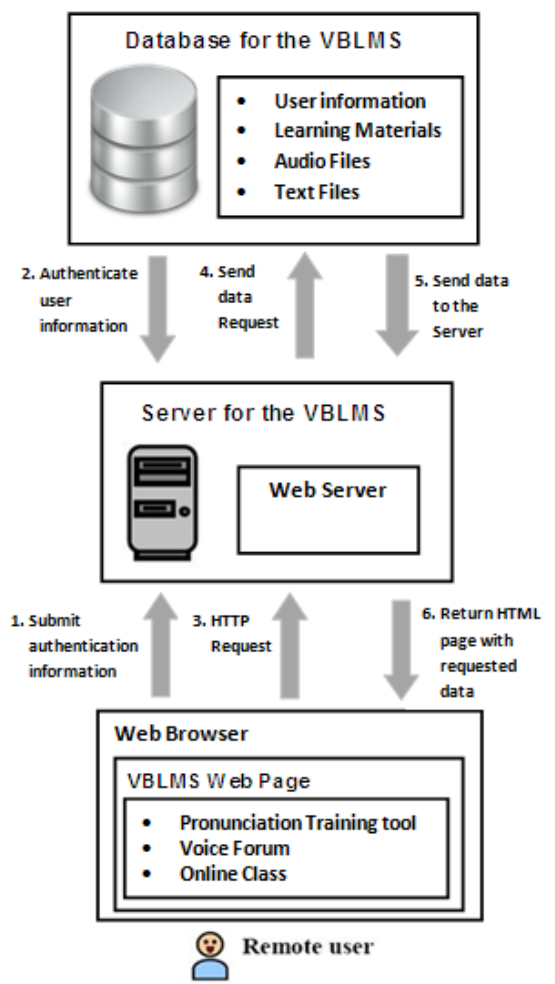

Figure 1. System Architecture

\section{System Implementation}

The base of this system is the Gong resource which can be used for the Web-based voice communication. At a basic level of usage, the system supports groups of people such as students and teachers participating in discussion groups using their computers. It supports both synchronous (realtime) and asynchronous voice and text communication.

A basic use of the Gong system is for people to participate and communicate in voice boards. A voice board is a collection of voice and/or text messages. Users can post a new message, and read or reply to any message on a board. This gong system has its own API. In order to develop the online voice based training tool, we will use that Gong API and 
we will make enhancements using JavaScript and VBScripts.

The Gong system provides web content developers the opportunity to add audio capability to a website. For example, a course web page can contain a Gong voice board which allows students to discuss with the teachers verbally on the Web. However one shortcoming of using a voice board is the lack of flexibility in web content design. For example, depending on different situations, teachers may want to have the ability to just play or stop an audio recording in a web page, without using or seeing any voice board. For this purpose there is a real-time communication method for web pages to communicate with the Gong applet. The applet can be hidden from the web page without the user knowing it is present. Using this method a web page can be greatly enriched by the features offered by the Gong system.

\subsection{Pronunciation Training Tool}

The Pronunciation training tool checks the pronunciation in two ways. Firstly, using Microsoft Speech Application Programming Interface (SAPI) as the correct pronunciation and scoring against it. This is happening in real time. Secondly, getting the feed back of a teacher, who checks the pronunciation of the student and send the feedback. The whole flow of the pronunciation tool is shown in Figure 2.

The student pronounces the given word or sentence and the system capture the signal and send it to the database. At the same time, it sends it to the Gong server as well. The signal comparison component retrieves the signal from the database and compares it with the Microsoft SAPI and generates a score file. The generated text file is sent to the student as their feedback. The signal that is sent to the Gong server will be stored there until the online teacher requests for it. When the teacher requests it, the teacher can listen to it and provide feedback to the student's e-mail account as an e-mail.

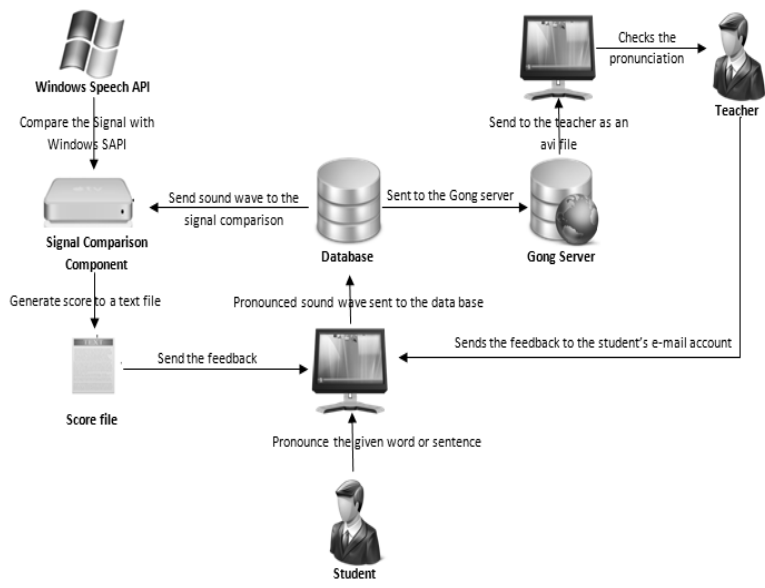

Figure 2. Pronunciation practicing by the help of the Microsoft Speech API and the teacher

\subsection{Voice Forum}

Using the voice forum, people can exchange their knowledge and ideas by using voice as the medium instead of text. Also people can search for a keyword using the search functionality and can listen to those ongoing or recorded discussions with that keyword. When two people are discussing, the voice get saved to the Gong Database. The same discussion is converted into text format using the "Voice to Text" component, and is saved as a text file in the Gong Database with the same file name as the voice file. When a user searches for a keyword, the application will search the content of all the text files for that keyword and sends the result to the user. So he can listen to those discussions with the keyword he wanted (see Figure 3).

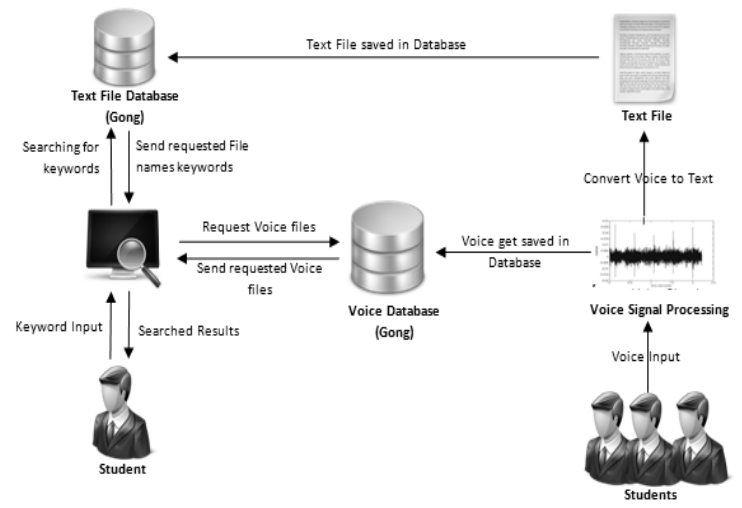

Figure 3. Voice discussion process

\subsection{Online Class Room}

This is a class where teachers and students interact over the internet. The idea is to build a central environment for the teachers and students to get together online and share their knowledge and ideas. This could be of two types, with participants engaging in activities simultaneously, which is referred to as 'synchronous' type, and the other category with participants engaging in real-time activities but the responses from each other are in a regular order which can be called ' asynchronous'.

\section{i. Online Language Learning Room -} Asynchronous: Students who have already logged in to the VBLMS can access a Gong voice board and start a discussion using text or else using his own voice. Then his voice track is passed to the gong server and the others can listen to this discussion by accessing the same Gong voice board and participate in the discussion as well. A typical scenario is as follows (see Figure 4);

1. Students log on to the VBLMS using his username and passwords.

2. Access Gong Voice Boards available in the VBLMS. 
3. Compose an idea/discussion and add to the Gong Voice Board. The data passes to the Gong server and is stored there.

The tutor logs on to the VBLMS system using his username and password, accesses the Gong Voice Boards available in the VBLMS, retrieves the voice tracks from the Gong server and sends replies to each student.

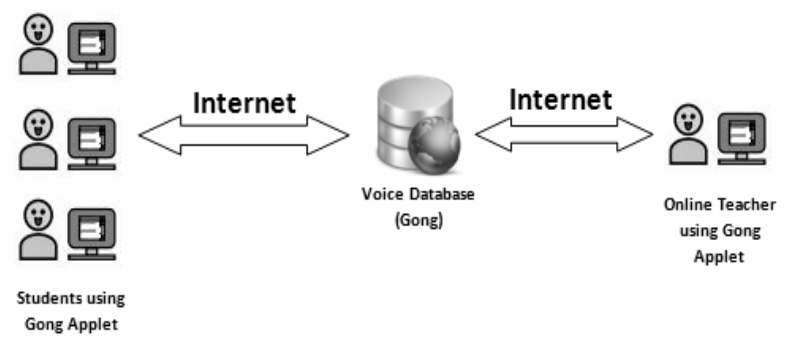

Figure 4. Online (Asynchronous) Language learning process

ii. Online Language Learning Room Synchronous: Tutors who have already logged on to the VBLMS can schedule an online Learning room there and invite the students to join the classroom synchronously. Unlike a normal virtual class room, here they can communicate live, text chat, and even audio and video conferencing can be done. Also, an Interactive White-board with multiple tabs is also available. (See Figure 5)

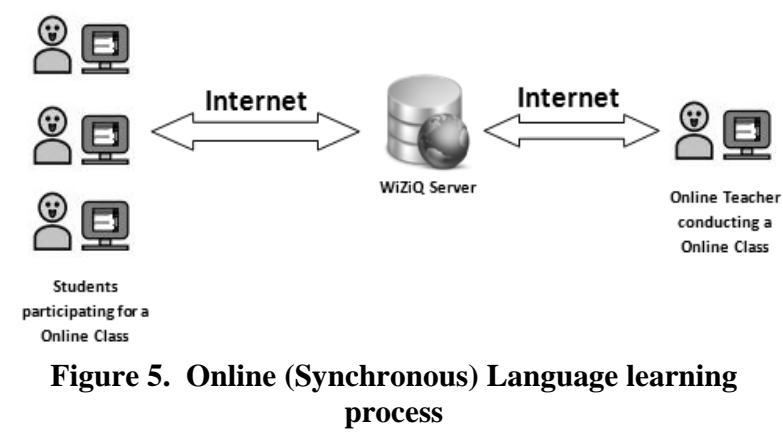

\section{User Evaluation}

After the implementation, we carried out a pilot study to find out the feasibility of this system in real time. The methods that we followed during the evaluation are described as follows.

Initially, as a pilot study, we identified the problems that can occur in the real user environment. We launched the system in an academic intranet and conducted a 1hour online class session with a few students. We did it using audio mode, therefore during the session the participants only heard the tutor's voice. Just before the class session, we gave them a questionnaire to get their feedback and ideas about the Online Language Learning Environment.

During the session, 2 of our members were facing some technical difficulties; they did not hear the tutor's voice very clearly and fluidly. So we marked that point to avoid that sort of difficulties during our next session and also came up with the following decisions.

- We need to have good internet connection during the session. Especially the tutor should have a speedy internet connection.

- Flash Player should be installed in every client machine.

After the session, we gave the participants another questionnaire to get their ideas about the session to prepare for our next evaluation.

Just after fixing the technical difficulties that arose during the pilot study, we tried to do a real academic lecture using this virtual language class room. For this purpose we invited a skilled communication tutor to do a lecture online by the help of the VBLMS.

Prior to this event, we had to make some minor adjustments in our approach and preparation. First there were few mistakes in our questionnaire, we had to correct those mistakes and improve the quality of the questionnaire by adding new questions so that it covers every important aspect.

Another problem we had in our pilot test was that some students had difficulty listening to the tutor's voice, because there were some problems in the internet ports and audio devices. By introducing a higher network bandwidth we were able to fix the problem and the students were able to interact with the tutor by using the whiteboard, chat and the microphone.

\subsection{Lecture on Virtual Language Class}

Afterwards, we handed out the questionnaire to the students. The questionnaire consisted of two parts;

- Before attending the Virtual class

- After attending the Virtual class

We asked the students to complete the 1st questionnaire prior to the commencement of the virtual class. Then we asked the tutor to log onto the system. Then he sent the link of the "virtual class" to the email accounts of the students. The students logged on to the VBLMS and started to interact with the tutor. They used the white board, chat and also voice to interact. This time they were able to see the lecturer at the top of the screen, because he had used a web camera for the session. In this instance, the class progressed smoothly without having any interruption. At the end of the lecture, we asked students to fill out the second part of the questionnaire. 


\subsection{Results}

For the virtual class session, twenty two (22) students attended, and their ideas and feedback about the class is described as follows;

- Participants expect to share knowledge and ideas, get proper guidance from a professional tutor as well as get a new experience from a virtual class room.

- It helps to keep away the shyness of participants during discussions, unlike in a traditional classroom.

- People are looking forward to feel the experience of such a tool as VBLMS because it saves their time and removes the geography barriers.

- Most of the participants feel comfortable with the virtual class sessions and they like to do their tasks with the help of the others as well.

- Most of them prefer to learn from a tutor rather than reading written instructions; they prefer spoken and visual presentations rather than reading text and assignments.

\section{Conclusion}

The goals of this Paper is to share the knowledge about the research project we have carried out to enhance the English pronunciation skill of a humans, develop the effectiveness of the way people communicate, the way people exchange their ideas with each other through the web using their own voice and to support distance learning and e-learning by using the voice as the medium.

During our research, we considered about the above facts, and we proposed to design the "Online Voice Based Learning Environment for English Language". We proposed three main components to be available in this environment.

i. Pronunciation training tool - Build an online system which can assist people to improve their pronunciation.

ii. Voice forum - Build an online voice based forum to share the knowledge among each other. It would also allow the keyword searching on previous discussions as well.

iii. Online Language learning room - Build an "Online Class Room" which is based on voice format. Not like traditional Learning Material System (LMS), the people will able to join online classes get the help of online mentors and share the knowledge.

Through this section we supposed to look around, every aspect of our research and find out the limitation, drawbacks and the solutions for those things as future works, etc.

There are few standalone applications which are used commercially in courses like TOFEL and IELTS to train proper pronunciation. However, there are no web based applications. If our solution was entirely a web solution that would be a value added enhancement for those applications.

Also in our system, we use the Microsoft Speech API for voice recognition. The Microsoft Speech API is based on US English. So if we are using this for British English based courses like IELTS that would be a problem. In the proposed system, the students will get the feedback from both the teacher and the system. Therefore in a British English based course, the student will get an accurate feedback only from the teacher. This will solve the API based problem.

In the concept of the Voice Forum, there are a few issues related to keyword search part. In this component, all the conversations are converting in to text format using a voice to text component. All the conversations are saved in both voice and text formats. When someone searches for a keyword, system will search in the text files for that keyword. It will list the topics which are related to resulting text files. However, the problem is that the system will save all the wanted an unwanted speeches in text format. A person who has bad pronunciation might enforce the system to maintain a text file with lot of garbage words. It will mislead the search. Also, the other problem is that all the speech words will not be recorded because of the bad or inconsistent pronunciations. So it will miss these keywords in the search.

Considering the Online classroom it's hard to simulate the real world classroom exactly. There are some limitations too. Key question is whether the cyber class would replace real classroom experience. Will it give the real classroom exposure? , should have to consider more to minimize the gap, the differences between those two in future. Some of the difficulties that around virtual classroom can be listed as below;

- Some of the communication problems faced by the participants were difficulties in explaining and understanding of the learning things.

- Through online asynchronous discussion, availability of sufficient computer facilities, and technical problems related to the computer server such as unavailability and problematic speed of network connections, may add minus points to the efficient online classes.

- Difficulties in uploading and downloading of files. Some students may not able to upload and download the learning materials because of the authentication problems or the browser used by the students may not compatible with the online system. 
- Majority of them would like to prepare themselves with the basic computer skills, such as "Microsoft Word," "Excel," "Equation Editor," and graphic software in order to follow the course effectively.

When it is compared with the real environment it is hard to imagine the moods and expressions of the user. If the system can use web camera in the conversations, it will solve this problem. However, it will not fill the gap completely. Somehow, it can limit this issue to some extent.

Through this project we have integrated several tools together and implement to work as a central portal. When we were integrating these open source tools we have come across with several problems.

- We have used Microsoft Vista SAPI as our speech recognition tool and because of that our whole project runs on Vista platform. Therefore, we have to integrate each and every open source components we have used during our project that supports with the Vista platform. We have to take a huge effort when we integrated those tools with the Vista.

- When connecting the Gong module with the Gong server, it did not work as it was written in the user manual. Since we are using Vista as our platform, the connection between the Gong server and Gong Moodle module will not establish as usual. We did modifications to establish that connection in our project. We manually edited the Gong.ini files to bridge the connection between the server and the Gong Moodle module. It took some time than we count out in our project schedule.

- Nanogong module which we have used for our voice forum component did not support for the Moodle version that we have used during the project. Since Nanogong module is compatible with the previous Moodle versions, we have to manually edit the code of the Nanogong module and had upgraded it to the current Moodle version which we have used in our project, to get full work of it.

\section{Acknowledgments}

We would like to thank Gibson Lam, who is a specialist in programming. He provided us great information and helped behind us to correct most of our issues related to the project. And also the Moodle community, they have helped us lot by giving their expertise knowledge to our project problems.

\section{References}

[1] Australasian Journal of Educational Technology 2006, 22(3), 375-397, Available: http://www.ascilite.org.au /ajet/ajet22/tsai.html, Access date: November 2009

[2] M.E. Butler-Pascoe, \& K.M. Wiburg, Technology and teaching English language learners, MA: Pearson Education, Inc., 2003.

[3] John M. Vitto, Relationship-driven Classroom Management: Strategies that Promote Student Motivation. Corwin Press, 2003.

[4] J. Leather, Second-language speech research: An introduction, Language Learning, 49(1). 1999, 1-56.

[5] M.J. Munro, Pronunciation: What are the Expectations?. A primer on accent discrimination in the Canadian context. TESL Canada Journal, Vol. IV, No. 1, 200338-51, 1998.

[6] D. Newby, Mediating between theory and practice in the context of different learning cultures and languages, European Centre for Modern Languages. Council of Europe Publishing, Graz, 2003.

[7] T. Pi-Hua, Bridging pedagogy and technology: User evaluation of pronunciation oriented CALL software. Australasian Journal of Educational Technology, vol. 22(3), pp. 375-397, 2006.

[8] Tsai Pi-Hua, 'Bridging pedagogy and technology: User evaluation of pronunciation oriented CALL software', Australasian Journal of Educational Technology, Vol 22(3), pp. 375-397, viewed 30 December 2009, http://www.ascilite.org.au/ajet/ajet22/tsai.html.

[9] L. Volle, Analyzing Oral Skills in Voice E-Mail and Online Interviews, Language Learning \& Technology, vol. 9, no. 3, pp. 146-163, 2005.

[10] WiZiQ Dashboard, http://www.wiziq.com/tour/LearnTeach-live-Virtual-Classroom.aspx,Access date: December 2009

[11] Egbert, J., Review of connected speech. Language Learning \& Technology, January 2004, Volume 8, Number 1, pp. 24-28

[12] Egbert, J., T. Paulus, \& Y. Nakamichi (2002), The impact of CALL instruction on classroom computer use: A foundation for rethinking

technology in teacher education. Language Learning \& Technology, September 2002, Volume 6, Number 3, pp. 108-126

[13] Neri, A., C. Cucchiarinim \& H. Strick (2002), Feedback in Computer Assisted Pronunciation Training: When technology meets pedagogy, A2RT, Department of Languages and Speech University of Nijmegen, The Netherlands. 
International Journal of Digital Society (IJDS), Volume 2, Issue 1, March 2011

[14] Eskenazi, M. (1999), Using automatic speech processing for foreign language pronunciation tutoring: Some issues and a prototype.

Language Learning \& Technology, January 1999, Volume 2, Number 2 pp. 62-7

[15] Brown, Adam (Ed.), Approaches to Pronunciation Teaching. Hertfordshire: Phoenix ELT, 1992.

[16] Chun, D. (1998), Signal analysis software for teaching discourse intonation. Language Learning \& Technology, July 1998, Vol. 2, No. 1, pp. 74-93.

[17]http://www.forrester.com/rb/Research/add_virtual_clas sroom_to_online_learning 